\title{
Inverse Functions Define Series (Polylogarithm)
}

Benedict Irwin ${ }^{1}$

${ }^{1}$ University of Cambridge

November 2, 2020

\section{Introduction}

I am converting this answer from a post online

\section{Main}

For the Polylogarithm we have the series representation

$$
\operatorname{Li}_{s}(z)=\sum_{k=1}^{\infty} \frac{z^{k}}{k^{s}}
$$

if we perform a [series reversion][1] on this (term by term) we end up with an expansion for the inverse function

$$
\mathrm{Li}_{s}^{-1}(z)=\sum_{k=1}^{\infty} a_{k} z^{k}
$$

the first few coefficients are

$$
\begin{array}{r}
a_{1}=1 \\
a_{2}=-2^{-s} \\
a_{3}=2^{1-2 s}-3^{-s} \\
a_{4}=56^{-s}-8^{-s}\left(5+2^{s}\right)
\end{array}
$$

there may be a pattern in there somewhere, but the terms seem to grow quite large and complicated rather quickly. For some reason I considered looking at the inverse Mellin transform of these coefficients, multipled by a gamma function, we can denote these functions $e_{k}(x)$

$$
e_{k}(x)=\mathcal{M}^{-1}\left[\Gamma[s] a_{k}(s)\right](s)
$$

these begin

$$
\begin{array}{r}
e_{1}(x)=e^{-x} \\
e_{2}(x)=-e^{-2 x} \\
e_{3}(x)=2 e^{-4 x}-e^{-3 x} \\
e_{4}(x)=-5 e^{-8 x}+5 e^{-6 x}-e^{-4 x}
\end{array}
$$


in each term $e_{k}(x)$ there are $P(k-1)$ exponential functions, where $P(k)$ from $k=0$ goes like $1,1,2,3,5,7, \cdots$ and are the partition numbers [A000041][2]. The coefficients in this grid of exponentials goes like:

$$
\begin{array}{r}
\alpha_{1}=[+1] \\
\alpha_{2}=[-1] \\
\alpha_{3}=[-1,2] \\
\alpha_{4}=[-1,5,-5] \\
\alpha_{5}=[-1,6,3,-21,14] \\
\alpha_{6}=[-1,7,7,-28,-28,84,-42] \\
\alpha_{7}=[-1,8,8,4,-36,-72,-12,120,180,-330,132]
\end{array}
$$

, and appear to be given by [A111785][3]. Interestingly, the powers of the exponentials also appear to have a sequence, the terms go like [A074139][4], which is titled "Number of divisors of A036035(n).". A036035 is titled "Least integer of each prime signature, in graded (reflected or not) colexicographic order of exponents."

We can recreate a coefficient by performing the Mellin transform and dividing through by $\Gamma(s)$

$$
a_{k}(s)=\frac{1}{\Gamma(s)} \mathcal{M}\left[e_{k}(x)\right](s)=\frac{1}{\Gamma(s)} \int_{0}^{\infty} x^{s-1} e_{k}(x) d x
$$

Then we can write

$$
e_{k}(x)=\sum_{l=1}^{P(k-1)} \alpha_{k l} e^{-\beta_{k l} x}
$$

where $\beta_{k}$ are the anaolgous rows of A074139

$$
\begin{array}{r}
\beta_{1}=[1] \\
\beta_{2}=[2] \\
\beta_{3}=[3,4] \\
\beta_{4}=[4,6,8] \\
\beta_{5}=[5,8,9,12,16] \\
\beta_{6}=[6,10,12,16,18,24,32]
\end{array}
$$

we we know then the Mellin transform of $a e^{-b x}=a b^{-s} \Gamma(s)$, and it's as simple as, grouping the terms which are now understood

$$
\begin{gathered}
a_{k}(s)=\frac{1}{\Gamma(s)} \int_{0}^{\infty} x^{s-1} \sum_{l=1}^{P(k-1)} \alpha_{k l} e^{-\beta_{k l} x} d x \\
a_{k}(s)=\sum_{l=1}^{P(k-1)} \frac{1}{\Gamma(s)} \int_{0}^{\infty} x^{s-1} \alpha_{k l} e^{-\beta_{k l} x} d x \\
a_{k}(s)=\sum_{l=1}^{P(k-1)} \frac{1}{\Gamma(s)} \alpha_{k l} \beta_{k l}^{-s} \Gamma(s) \\
a_{k}(s)=\sum_{l=1}^{P(k-1)} \alpha_{k l} \beta_{k l}^{-s} \\
\operatorname{Li}_{s}^{-1}(z)=\sum_{k=1}^{\infty} a_{k}(s) z^{k}=\sum_{k=1}^{\infty} \sum_{l=1}^{P(k-1)} \alpha_{k l} \beta_{k l}^{-s} z^{k}
\end{gathered}
$$


the limitation here is understanding where the terms in the sequences referenced come from, and finding whether any tractable forms exist for $\alpha$ and $\beta$. We already know that $\beta_{k l}=\sigma_{0}\left(\gamma_{k l}\right)$ for the $\gamma$ in A036035, and divisor counting function $\sigma$.

$$
\mathrm{Li}_{s}^{-1}(z)=\sum_{k=1}^{\infty} \sum_{l=1}^{P(k-1)} \frac{\alpha_{k l} z^{k}}{\sigma\left(\gamma_{k l}\right)^{s}}
$$

\section{Transform and Duality}

In this sense we could define a transform between the divisor function and the polylogarithm

$$
T[\sigma(n)] \rightarrow \operatorname{Li}_{s}(z)
$$

we might ask, what other number-theory related functions transform to in this manner? The most interesting (for which there is an obvious pattern) seems to be for the Liouville lambda function,

$$
T[\lambda(n)] \rightarrow\left(z+z^{3}+z^{5}+z^{7}+z^{9}+z^{11}+\cdots\right)+(-1)^{s}\left(z^{2}+z^{4}+z^{6}+z^{8}+z^{10}+\cdots\right)=\frac{z+(-1)^{s} z^{2}}{1-z^{2}}
$$

it seems that

$$
\begin{gathered}
\operatorname{Inv}\left(z-z^{2}\right)=\sum_{k=1}^{\infty} \sum_{l=1}^{P(k-1)} \alpha_{k l} z^{k} \mu\left(\gamma_{k l}\right)=z \mathrm{GF}(\text { Catalan }) \\
\sum_{k=1}^{\infty} \sum_{l=1}^{P(k-1)} \alpha_{k l} z^{k-1} \Omega\left(\gamma_{k l}\right)=z \operatorname{altGF}(\text { Catalan })
\end{gathered}
$$

A nice result seems to be

$$
\begin{gathered}
\operatorname{Inv}(\sqrt{z} \operatorname{atanh}(\sqrt{z})))=\sum_{k=1}^{\infty} \sum_{l=1}^{P(k-1)} \frac{\alpha_{k l} z^{k}}{\sigma\left(\gamma_{k l}^{2}\right)} \\
\operatorname{Inv}\left(\frac{z}{n} \Phi\left(z, 1, \frac{1}{n}\right)\right)=\sum_{k=1}^{\infty} \sum_{l=1}^{P(k-1)} \frac{\alpha_{k l} z^{k}}{\sigma\left(\gamma_{k l}^{n}\right)}
\end{gathered}
$$

and in general

$$
\operatorname{Inv}\left(\frac{z}{n^{s}} \Phi\left(z, s, \frac{1}{n}\right)\right)=\sum_{k=1}^{\infty} \sum_{l=1}^{P(k-1)} \frac{\alpha_{k l} z^{k}}{\sigma\left(\gamma_{k l}^{n}\right)^{s}}
$$

Nice examples are

$$
\begin{gathered}
\sum_{k=1}^{\infty} \sum_{l=1}^{P(k-1)} \alpha_{k l} z^{k-1} \Omega\left(\gamma_{k l}\right)^{2}=\left(-z+4 z^{2}-9 z^{3}+16 z^{4}-\cdots\right)=A 007297 \\
\sum_{k=1}^{\infty} \sum_{l=1}^{P(k-1)} \alpha_{k l} z^{k-1} \Omega\left(\gamma_{k l}^{n}\right)^{s}=\left(-(n)^{s} z+(2 n)^{s} z^{2}-(3 n)^{s} z^{3}+(4 n)^{s} z^{4}-\cdots\right)
\end{gathered}
$$

